\title{
Effects of quercetin on kidney injury induced by doxorubicin
}

\author{
Yagmurca $\mathrm{M}^{1}$, Yasar $\mathrm{Z}^{2}$, Bas $\mathrm{O}^{3}$ \\ Department of Histology and Embryology, Faculty of Medicine, Turgut Özal University, Ankara, Turkey. \\ yagmurcamd@gmail.com
}

\begin{abstract}
OBJECTIVES: The anthracycline antitumor drug doxorubicine causes severe nephrotoxicity in a variety of experimental animals and may be nephrotoxic to humans. The aim of present study was to determine the protective effects of quercetin against doxorubicin-induced kidney injury with light microscopy.

METHODS: Forty male Wistar albino rats were divided into four groups: control, doxorubicin, doxorubicin + quercetin and quercetin. A single dose of $20 \mathrm{mg} / \mathrm{kg} / \mathrm{i}$.p. doxorubicin was used to induce injury. Quercetin was administrated orally against doxorubicin toxicity. The kidneys were examined under light microscopy after H\&E (hematoxylin-eosin) staining and the changes were scored.

RESULTS: Significant tissue injury was observed in doxorubicin-administered group. Among these injuries, renal tubular dilatation, tubular vacuolar changes, glomerular vacuolization, decrease in bowman space, bowman capsule thickening, and interstitial infiltration were evident. However, the injury induced by doxorubicin was attenuated with quercetin administration.

DISCUSSION: Quercetin decreased doxorubicin-induced kidneydamage (Tab. 1, Fig. 4, Ref. 27). Textin PDFwww.elis.sk. KEY WORDS: doxorubicin, quercetin, nephrotoxicity, rat.
\end{abstract}

\section{Introduction}

Doxorubicin is an anthracycline which has been used in the treatment of cancer; however, it has nephrotoxic effects. Oxygen free radicals are produced in larger amounts due to the use of agents like doxorubicin, and this leads to oxidative damage in cellular membranes, organelles and even in genetic material (1). The most important indicators of this damage are lipid peroxidation and protein oxidation. Antioxidant enzymes superoxide dismutase (SOD) and catalase (CAT) are endogenous resources which can prevent this damage through removal of oxygen radicals.

The oxidant-antioxidant system has different sources, one of which is nitric oxide (NO). NO can be both a scavenger and a producer of free oxygen radicals. NO reacts with superoxide anions and is converted into peroxynitrite. Thus, peroxynitrite does both removes superoxide anion and becomes a damage-producing radical. Another source of the oxidant system is malondialdehyde (MDA), an indicator of lipid peroxidation. Other sources of radicals include neutrophils and myeloperoxidase (MPO) system. MPO production increases in inflammatory responses and produces hypochlorite radicals leading to cellular damage (2).

${ }^{1}$ Department of Histology and Embryology, Faculty of Medicine, Turgut Özal University, Ankara, Turkey, ${ }^{2}$ Department of Histology and Embryology, Faculty of Medicine, Afyon Kocatepe University, Afyonkarahisar, Turkey, and ${ }^{3}$ Department of Anatomy, Faculty of Medicine, Ordu University, Ordu, Turkey Address for correspondence: M. Yagmurca, MD, Department of Histology and Embryology, Faculty of Medicine, Turgut Özal University, Ankara, Turkey.

Phone: +90.312.3977400, Fax: +90.312 .3977448$

Acknowledgement: This study was supported by Afyon Kocatepe University Scientific Research Projects Coordination Unit (Project Number: 08.TIP.01).
Flavonoid, plant pigments have been known for more than a century. Quercetin, a flavonol, is abundant in food, especially in onion. Tea is also rich in flavonol, particularly quercetin and kaempferol (3).

Recent studies have shown that flavonoids prevent DNA damage through certain mechanisms. They can be effective through protection and augmentation of endogenous oxidants. Most of flavonoids are able to activate glutathione-S-transferase (GST). Flavonoids including quercetin, myricetin and fisetin show their effects by significantly increasing GST activity (3-5).

The aim of this study was to investigate protective effects of quercetin against doxorubicin-induced renal injury under light microscope.

\section{Material and methods}

Forty male adult Wistar albino rats were used and divided into four groups: control (I; $\mathrm{n}=10$ ); doxorubicin (II; $\mathrm{n}=10$ ); doxorubicin + quercetin, (III; $\mathrm{n}=10)$; quercetin $(\mathrm{IV} ; \mathrm{n}=10)$. The control group received $0.5 \mathrm{ml} 0.9 \% \mathrm{NaCl}$ intraperitoneal (i.p.) and $1 \mathrm{cc}$ $0.9 \% \mathrm{NaCl}$ orally for 10 days. The animals in the doxorubicin group were treated with a single $20 \mathrm{mg} / \mathrm{kg} /$ i.p. dose of doxorubicin hydrochloride $(\mathrm{C} 27 \mathrm{H} 29 \mathrm{NO} 11 \mathrm{HCl})$ (Doxo Teva $50 \mathrm{mg}$ vial) on day 3 of the experiment. Group III received oral quercetin dihydrate (C15H10O7.2H20; Quercetin dihydrate, Sigma) $50 \mathrm{mg} /$ $\mathrm{kg}$ /day except on day 3 on which doxorubicin was applied. Group IV was administered oral quercetin dihydrate $\left(\mathrm{C} 15 \mathrm{H} 10 \mathrm{O} 7.2 \mathrm{H}_{2} 0\right.$; Quercetin dihydrate, Sigma) $50 \mathrm{mg} / \mathrm{kg} /$ day for 10 days. After administration of the drugs, the rats were decapitated and their kidneys were removed and fixed in $10 \%$ neutral-buffered formalin. Tissue samples were stained with H\&E (hematoxylin-eosin) and histologically examined under light microscope. 
Light microscopic changes as tubular necrosis, atrophy, glomerular injury, vascular congestion, thrombosis, and interstitial inflammation were scored as follows: normal histology as 0 points; minimal changes as 1 point; moderateinjury as 2 points; severeinjury as 3 points.

Data were analyzed using SPSS for Windows v. 13.0 (Chicago, Ill., USA). For assessment of the homogeneity of groups, ShapiroWilk test was used. The comparison among groups was made with Kruskal-Wallis H Test. Mann-Whitney U Test is used for pairwise comparison of groups. $\mathrm{p}<0.05$ was considered significant.

\section{Results}

The control group revealed normal renal histology (Fig. 1). Nephrotoxic tissue damage due to doxorubicin is demonstrated in Figure 2. There was tubular necrosis and atrophy, vacuolization, glomerular damage, vascular congestion-thrombosis, and interstitial damage in the renal tissue in the doxorubicin group. Tissue morphology was improved with quercetin administration in Group III (Fig. 3). Quercetin administration alone caused no apparent changes in renal tissue (Fig. 4). All renal damage indicators, except for interstitial inflammation, significantly decreased in doxorubicin+quercetin group compared to doxorubicin group $(\mathrm{p}<$ 0.05 ) (Tab. 1). In addition, doxorubicin and doxorubicin+quercetin

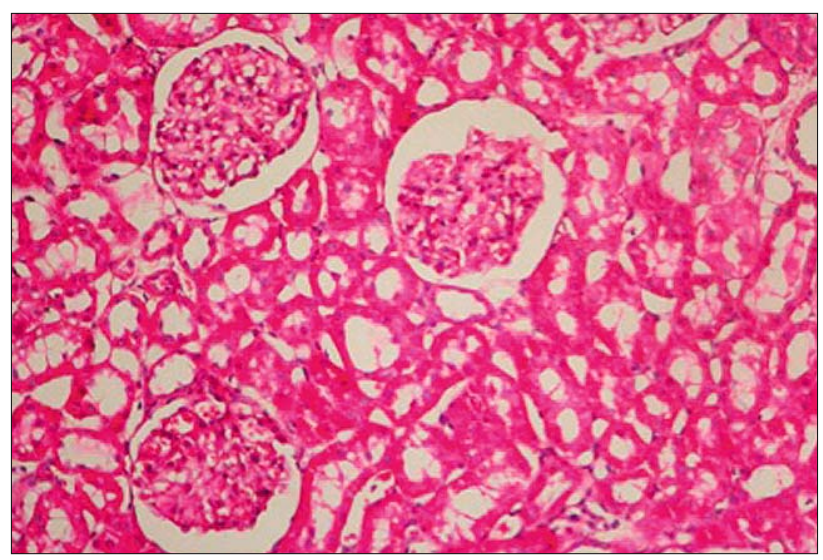

Fig. 1. Normal renal histology in control group.

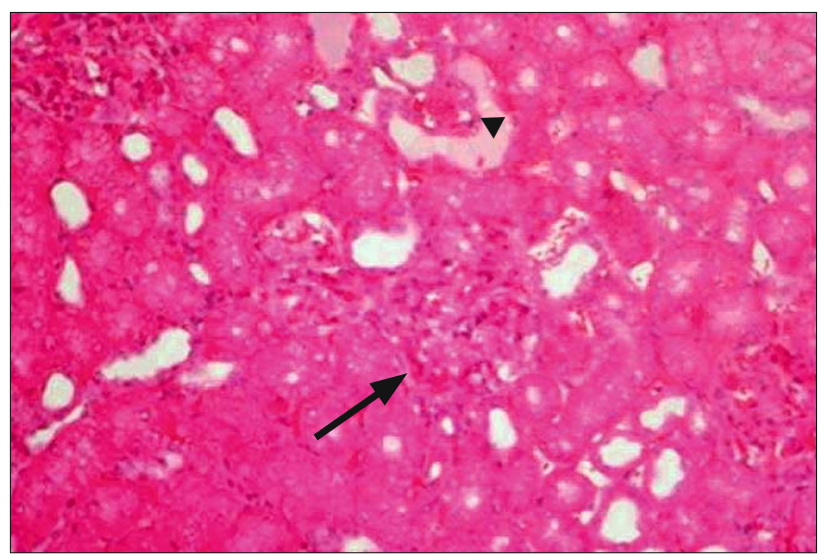

Fig. 2. Glomerular congestion (arrow) and eosinophylic material in a tubule (arrowhead) in Group II.

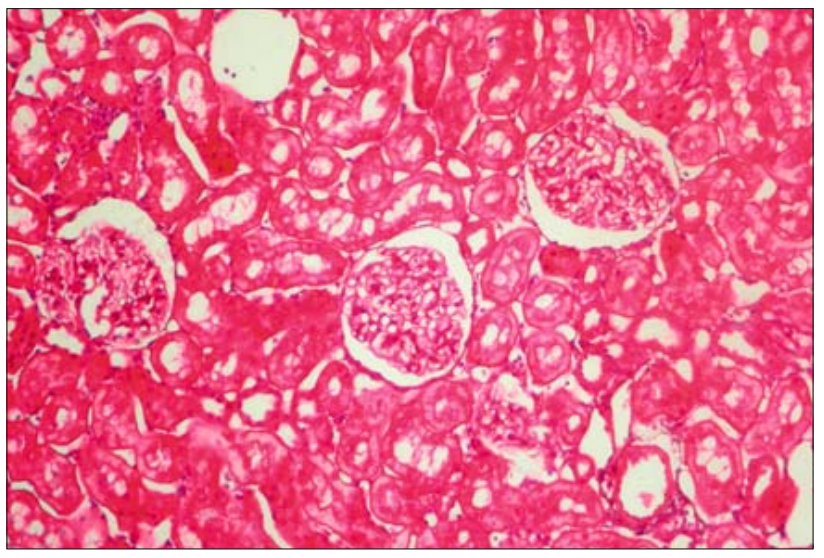

Fig. 3. Improved glomerular and tubular histology in Group III.

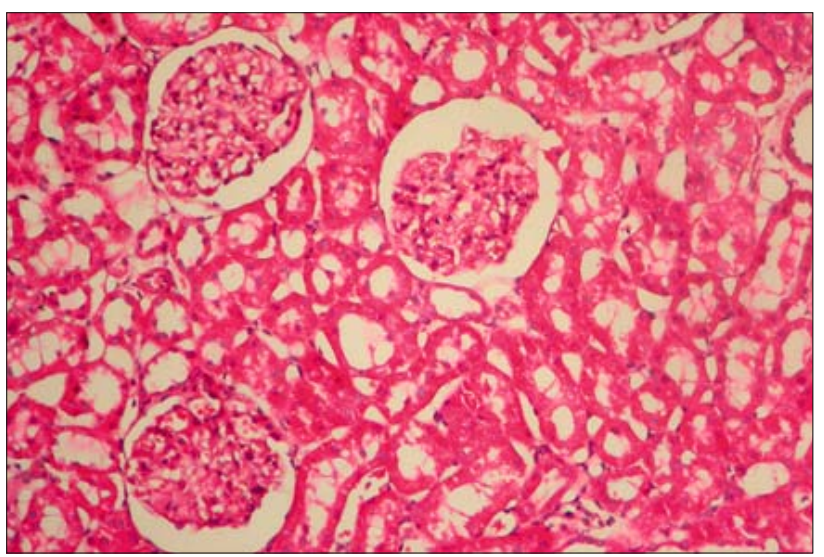

Fig. 4. Normal renal histology in Group IV.

groups yielded significantly increased pathological changes when compared to the control group ( $\mathrm{p}<0.05$ ).

However, there was no significant difference between quercetin group and control group $(\mathrm{p}>0.05)$, in terms of tissue damage (Tab. 1).

\section{Discussion}

Doxorubicin is used to treat solid tumors like carcinomas of the uterus, breasts, thyroid, esophagus, ovaries and lungs, acute leukemia, Hodgkin's and non-Hodgkin's lymphomas and soft tissue sarcomas $(6,7)$. Doxorubicin has been shown to cause renal toxicity due to oxidative stress in various experimental animal models and human studies $(8,9)$. As a result of oxygen use in aerobic organisms, reactive oxygen metabolites (ROM) occur naturally.

In a study by Liu et al, GSH, GST and SOD levels decreased in doxorubicin-administered rats (10). In a previous study conducted by our team, protective effects of caffeic acid phenethyl ester(CAPE) against doxorubicin-induced kidney injury were investigated. Histological examination showed glomerular vacuolization, tubular desquamation, adhesion, and thickening in Bowman's capsule and damage in the tubular and glomerular capillary basal membranes in doxorubicin group. Besides, the administration of CAPE combined with doxorubicin was observed to provide partial protection of the glomerules and tubules (11). Likewise, in the present study, we 
Tab. 1. Statistical comparison of histological findings. Values are given as mean \pm standard deviation.

\begin{tabular}{|c|c|c|c|c|c|}
\hline Groups & Tubular necrosis-atrophy & Tubular vacuolization & Glomerular injury & Vascular congestion-thrombosis & Interstitial inflammation \\
\hline $\bar{I}$ & $0.00 \pm 0.00$ & $0.00 \pm 0.00$ & $0.00 \pm 0.00$ & $0.00 \pm 0.00$ & $0.00 \pm 0.00$ \\
\hline II & $2.20 \pm 0.13$ & $2.50 \pm 0.16$ & $2.20 \pm 0.13$ & $2.00 \pm 0.21$ & $1.80 \pm 0.20$ \\
\hline III & $1.40 \pm 0.16$ & $1.40 \pm 0.16$ & $1.40 \pm 0.16$ & $1.20 \pm 0.13$ & $1.30 \pm 0.15$ \\
\hline IV & $0.20 \pm 0.13$ & $0.30 \pm 0.15$ & $0.3 \pm 0.15$ & $0.20 \pm 0.13$ & $0.20 \pm 0.13$ \\
\hline \multicolumn{6}{|l|}{$\mathrm{p}$ value } \\
\hline I and II & 0.000 & 0.000 & 0.000 & 0.000 & 0.000 \\
\hline I and III & 0.000 & 0.000 & 0.000 & 0.000 & 0.000 \\
\hline II and III & 0.009 & 0.002 & 0.009 & 0.015 & n.s. \\
\hline I and IV & n.s. & n.s. & n.s. & n.s. & n.s. \\
\hline
\end{tabular}

Group I: Control, Group II: Doxorubicin, Group III: Doxorubicin+Quercetin, Group IV: Quercetin

n.s.: not significant

administered a single dose of doxorubicin $20 \mathrm{mg} / \mathrm{kg}$ into the peritoneum which resulted in similar tubular and glomerular damage.

Histological effects of doxorubicin nephrotoxicity were also shown by Jadhav et al (12). In another study by Lenderink et al., rats aged 8-10 weeks were administered a single dose of doxorubicin $10-11 \mathrm{mg} / \mathrm{kg}$ and sacrificed $4-5$ weeks later. They observed glomerular sclerosis, tubular dilatation, and tubulointerstitial inflammation upon morphological examinations (12).

Weening et al reported that the rats administered with a single intravenous dose of doxorubicin $7.5 \mathrm{mg} / \mathrm{kg}$ had proteinuria at a mean amount of $98 \mathrm{mg}$, swelling in the glomerular epithelial cells, obliteration and vacuolization on morphological examinations (13). However, in the present study, we detected more severe glomerular and tubular damage with doxorubicin $20 \mathrm{mg} / \mathrm{kg}$. The severity of histological injury may be attributable to the higher doses of doxorubicin in our study.

In a study by Dziegiel et al, acute toxicity occurred after administering a single intravenous dose of doxorubicin or daunorubicin. Additionally, subchronic toxicity was created by 3 doses $(3 \mathrm{mg} / \mathrm{kg}$ ) of the same antibiotics administered in one-week intervals. They observed glomerular sclerosis, glomerular vacuolization, tubular dilatation, tubular atrophy, interstitial lymphocytic infiltration, and material rich in protein in the tubular lumen in rats developing subchronic toxicity (14). In addition to the changes reported in the study by Dziegiel et al, in the present study we detected a reduction in Bowman's space and thickening in Bowman's capsule. This can be attributed to the dose we administered.

In a study by Erdogan et al, $0.6 \mathrm{mg} / \mathrm{kg} /$ day doxorubicin was administered to New Zealand rabbits for 6 days, while blood specimens were taken two hours after each administration, and histopathological examinations were performed. Doxorubicin-administered rabbits were observed to have increased serum urea and creatinine. In line with our report, their histopathological examination revealed degenerated tubular epithelium, large intertubular bleeding areas, glomerular atrophy, enlarged glomerular space, and material rich in protein in the tubular lumen (15).

In a study by El-Sheikh et al, doxorubicin caused dilatation in Bowman's space and marked degeneration of renal tubules that showed exfoliated cells, protein casts, and cystic dilatation (16).

Saad et al evaluated the effects of doxorubicin $25 \mathrm{mg} / \mathrm{kg}$ administration on heart, liver, and kidney tissues using biochemical and histopathological parameters and showed that focal tubular atrophy developed in the renal sections in doxorubicin-administered rats (17).
Boonsanit et al showed that intravenous administration of doxorubicin $7.5 \mathrm{mg} / \mathrm{kg}$ created nephrotoxic syndrome based on biochemical and histopathological parameters. They demonstrated glomerular capillary dilatation and tubular dilatation in sections of renal specimens (18).

In a study by Park et al., the rats were administered with a single dose of doxorubicin $7.5 \mathrm{mg} / \mathrm{kg}$ and sacrificed 21 hours later. Tubular dilatation, eosinophilic material in the tubular lumen, glomerular vacuolization, and thickening and adhesion in the Bowman's capsule were identified (19). In the present study, we also observed similar renal damage following an administration of a single dose of doxorubicin $20 \mathrm{mg} / \mathrm{kg}$.

In the study by Mansour et al., an increase in serum urea levels and a sharp decrease in total protein and albumin levels were observed 24-48 hours after a single dose of doxorubicin $15 \mathrm{mg} /$ $\mathrm{kg}$ had been administered. An increase in lipid peroxidation was also observed 24 hours after administration of doxorubicin. They claimed that captopril could be protective against nephrotoxicity created by doxorubicin (20). In a similar study by Bertani et al, proteinuria was detected 4-5 days after i.p. injection of a single dose of doxorubicin $7.5 \mathrm{mg} / \mathrm{kg}(21)$.

Flavonoids belong to polyphonic compounds and can be found in all plants. Quercetin is one of the flavonoids known to have antioxidant features and achieve oxygen free radical uptake. Tea is rich in quercetin and kaempferol, which belong to flavonols and flavones (22).

Since quercetin was shown to have an antioxidant effect, we attempted to confirm the effect of quercetin in different doses on the damage created by doxorubicin. In literature, the protective effects of quercetin against different toxic agents were shown.

Behling et al administered a single dose of cisplatin $5 \mathrm{mg} / \mathrm{kg}$ into the peritoneum and gave quercetin $50 \mathrm{mg} / \mathrm{kg}$ through gavage 24 hours before administration of cisplatin. The administration of cisplatin was continued for 2, 5, and 20 days. They reported that cisplatin increased lipid peroxidation, urine volume, and plasma creatinine levels, while decreasing urine osmolality. However, those findings were reported to be reduced with quercetin (23).

Bongiovanni et al. used the plant flavonoids silymarin and quercetin to eliminate cytotoxicity caused by arsenite and made a conclusion that flavonoids taken through diet can be protective against this toxicity (24).

In a study by Ikizler et al, the protective effects of quercetin given as chronic treatment and acute infusion on ischemia-reperfusion were investigated. Final hemodynamic and biochemical pa- 
rameters indicated a myocardial improvement in all stages of reperfusion in the quercetin group compared to the control group (25).

Polat et al investigated effects of intramuscular administrations of quercetin $50 \mathrm{mg} / \mathrm{kg}$ and desferrioxamine $100 \mathrm{mg} / \mathrm{kg}$ on renal damage created through hepatic ischemia-reperfusion. It was found that quercetin and desferrioxamine provided beneficial effects through modification of glutathione and MDA levels (26). In a similar study by Tokyol et al, reperfusion was created by $45-$ min hepatic ischemia, and the effects of intramuscular administrations of quercetin $50 \mathrm{mg} / \mathrm{kg}$ and desferrioxamine $100 \mathrm{mg} / \mathrm{kg}$ on liver damage were investigated. Plasma alanine aminotransferase levels, and MDA and glutathione activities were measured, and histopathological examinations of the obtained liver tissues were made. It was concluded that quercetin and desferrioxamine had a protective effect on the liver tissue against ischemia-reperfusion injury (27).

\section{Conclusion}

It can be concluded that in the light of histological findings, quercetin can have beneficial therapeutic effects in terms of prevention of renal damage caused by doxorubicin. However, detailed further studies with lager series are needed to confirm this conclusion.

\section{References}

1. Funk F, Krüger K, Henninger C, Wätjen W, Proksch P, Thomale J, Fritz G. Spongean alkaloids protect rat kidney cells against cisplatin-induced cytotoxicity. Anticancer Drugs. 2014. doi:10.1097/CAD.0000000000000119.

2. Gurel A, Armutcu F, Sahin S, Sogut S, Ozyurt H, Gulec M, Kutlu NO, Akyol O. Protective role of alpha-tocopherol and caffeic acid phenethyl ester on ischemia-reperfusion injury via nitric oxide and myeloperoxidase in rat kidneys. Clin Chim Acta 2004; 339 (1-2): 33-41.

3. Staedler D, Idrizi E, Kenzaoui BH, Juillerat-Jeanneret L. Drug combinations with quercetin: doxorubicin plus quercetin in human breast cancer cells. Cancer Chemother Pharmacol 2011; 68 (5): 1161-1172.

4. Prakash D, Gopinath K, Sudhandiran G. Fisetin enhances behavioral performances and attenuates reactive gliosis and inflammation during aluminum chloride-induced neurotoxicity. Neuromol Med 2013; 15 (1): 192-208.

5. Bertani T, Poggi A, Pozzoni R. Adriamycin-Induced Nephrotic Syndrome in Rats: Sequence of Pathologic Events. Lab Invest 1982; 46 (1): 16-23.

6. Gokcimen A, Cim A, Tola HT, Bayram D, Kocak A, Ozgüner F, Ayata A. Protective Effect of N-Acetylcysteine, Cafeic Acid and Vitamin E on Doxorubicin Hepatotoxicity. Hum Exp Toxicol 2007; 26: 519-525.

7. Wang X, Teng Z, Wang H, Wang C, Liu Y, Tang Y, Wu J, Sun J, Wang $\mathbf{H}$, Wang J, Lu G. Increasing the cytotoxicity of doxorubicin in breast cancer MCF-7 cells with multidrug resistance using a mesoporous silica nanoparticle drug delivery system. Int J Clin Exp Pathol 2014; 7 (4): 1337-1347.

8. Injac R, Boskovic M, Perse M, Koprivec-Furlan E, Cerar A, Djordjevic A, Strukelj B. Acute Doxorubicin Nephrotoxicity in Rats with Malignant Neoplasm can be Successfully Treated with Fullerenol C60 (OH)24 via Suppression of Oxidative Stress. Pharmacol Rep 2008; 60 (5): 742-749.

9. Saenko IuV, Shutov AM, Napalkova SM, Selivanova OS, Evstegneeva EIu. The Effect of Erythropoetin on Doxorubicin-Induced Oxidative Stress in rat Kidney. Eksp Klin Farmakol 2005; 68 (6): 52-54
10. Liu LL, Li QX, Xia L, Li J, Shao L. Differential Effects of Dihydropyridine Calcium Antagonists on Doxorubicin-Induced Nephrotoxicity in Rats. Toxicology 2007; 231 (1): 81-90.

11. Yagmurca M, Erdogan H, Iraz M, Songur A, Ucar M, Fadillioglu E. Caffeic Acid Phenethyl Ester as a Protective Agent against Doxorubicin Nephrotoxicity in Rats. Clin Chim Acta 2004; 348: 27-34.

12. Jadhav VB, Thakare VN, Suralkar AA, Naik SR. Ameliorative effect of Luffa acutangula Roxb. on doxorubicin induced cardiac and nephrotoxicity in mice. Indian J Exp Biol 2013; 51 (2): 149-156.

13. Weening JJ, Rennke GH. Glomerular Permeability and Polyanion in Adriamycin Nephrosis in the Rat. Kidney Internat 1983; 24: 152-159.

14. Dziegiel P1, Suder E, Surowiak P, Jethon Z, Rabczyński J, Januszewska L, Sopel M, Zabel M. Role of exogenous melatonin in reducing the nephrotoxic effect of daunorubicin and doxorubicin in the rat. J Pineal Res 2002; 33 (2): 95-100.

15. Erdogan HM, Citil M, Tuzcu M. The Effect of L- Carnitine Administration on Doxorubicine Induced Hepatoxicity and Nephrotoxicity in Rabbits. Kafkas Univ Vet Fak Derg 2009; 15 (5): 733-738.

16. El-Sheikh AA1, Morsy MA, Mahmoud MM, Rifaai RA, Abdelrahman AM. Effect of coenzyme-q10 on Doxorubicin-induced nephrotoxicity in rats. Adv Pharmacol Sci 2012; 2012: 981461. doi:10.1155/2012/981461.

17. Saad SY, Najjar TA, Al-Rikabi AC. The Preventive Role Of Deferoxamine Against Acute Doxorubisin - Induced Cardiac, Renal and Hepatic Toxicity in Rats Pharmacol Res 2001; 43 (3): 211-218.

18. Boonsanit D, Kanchanapangka S, Buranakarl C. L-carnitine Ameliorates Doxorubicin-Induced Nephrotic Syndrome in Rats. Nephrology (Carlton) 2006; 11 (6): 569.

19. Park ES, Kim SD, Lee MH, Lee HS, Lee IS, Sung JK, Yoon YS. Protective Effect of N-acetylcysteine and Selenium against Doxorubicin Toxicity in Rats. J Vet Sci 2003; 4 (2): 129-136.

20. Mansour MA, El-Kashef HA, Al-Shabanah OA. Effect of Captopril on Doxorubicin-Induced Nephrotoxicity in Normal Rats. Pharmacol Res 1999; 39 (3): 233-237.

21. Bertani T, Poggi A, Pozzoni R. Adriamycin-Induced Nephrotic Syndrome in Rats: Sequence of Pathologic Events. Lab Invest 1982; 46 (1): 16-23.

22. Sak K. Site-specific anticancer effects of dietary flavonoid quercetin. Nutr Cancer 2014; 66 (2): 177-193.

23. Behling EB, Sendão MC, Francescato HD, Antunes LM, Costa RS, Bianchi Mde L. Comparative study of multiple dosage of quercetin against cisplatin-induced nephrotoxicity and oxidative stress in rat kidneys. Pharmacol Rep 2006; 58 (4): 526-532.

24. Bongiovanni GA, Soria EA, Ernard AR. Effects of the Plant Flavonoids Silymarin and Quercetin on Arsenite-İnduced Oxidative Stress in CHO-K1 Cells. Food ChemToxicol 2007; 45: 971-976.

25. Ikizler M, Erkasap N, Dernek S, Kural T, Kaygisiz Z. Dietary polyphenol quercetin protects rat hearts during reperfusion: enhanced antioxidant capacity with chronic treatment. Anadolu Kardiyol Derg 2007; 7 (4): 404-410.

26. Polat C, Tokyol C, Kahraman A, Sabuncuoglu B, Yilmaz S. The effects of desferrioxamine and quercetin on hepatic ischemia-reperfusion induced renal disturbance. Prostaglandins Leukot Essent Fatty Acids 2006; 74 (6): 379-383.

27. Tokyol C, Yilmaz S, Kahraman A, Cakar H, Polat C. The effects of desferrioxamine and quercetin on liver injury induced by hepatic ischaemiareperfusion in rats. Acta Chir Belg 2006; 106 (1): 68-72.

Received July 5, 2014. Accepted March 8, 2015. 